\title{
ESTIMATES FOR FRACTIONAL POWERS AND LOGARITHM OF OPERATORS WITH HILBERT-SCHMIDT RESOLVENTS AND PERTURBATION RESULTS
}

\author{
Michael Gil' \\ Department of Mathematics \\ Ben Gurion University of the Negev \\ P.0. Box 653, Beer-Sheva 84105, ISRAEL
}

\begin{abstract}
Let $A$ be a closed operator on a separable Hilbert space with the spectrum in the open right half-plane and a bounded Hermitian component, and let the resolvent of $A$ be a Hilbert-Schmidt operator. The paper deals with the function

$$
h_{\mu}(A)=\int_{0}^{\infty}(A+t I)^{-1} d \mu(t)
$$

where $\mu$ is a nondecreasing function and $I$ is the unit operator. We establish norm estimates and perturbations results for $h_{\mu}(A)$. As particular cases the fractional powers and logarithm of $A$ are considered.
\end{abstract}

AMS Subject Classification: 47A56, 47A63, 47A55

Key Words: Hilbert space; fractional power of operators; operator logarithm Hirsch type functions; perturbations

\section{Introduction and statement of the main result}

Let $\mathcal{H}$ be a separable complex Hilbert space with a scalar product $\langle.,$.$\rangle , the$ norm $\|\|=.\sqrt{\langle., .\rangle}$ and unit operator $I$. For a linear operator $T, D(T)$ is the domain, $\sigma(T)$ denotes the spectrum, $R_{z}(T)=(T-z I)^{-1} \quad(z \notin \sigma(T))$ is the resolvent, $T^{*}$ is the adjoint operator. If $T$ is bounded, then $\|T\|$ is its operator norm. By $\mathcal{S}_{p}(1 \leq p<\infty)$ we denote the Schatten - von Neumann ideal of 
compact operators $K$ with the finite norm $N_{p}(K):=\left[\operatorname{trace}\left(K K^{*}\right)^{p / 2}\right]^{1 / p}$. So $\mathcal{S}_{2}$ is the Hilbert-Schmidt ideal.

Throughout the paper, $A$ and $\tilde{A}$ are closed linear operators on $\mathcal{H}$. The paper is devoted to the norm estimates for operator valued functions of the form

$$
h_{\mu}(A)=\int_{0}^{\infty}(A+t I)^{-1} d \mu(t)
$$

where $\mu(t)$ is a nondecreasing function defined on $[0, \infty)$ and satisfying the condition

$$
\int_{0}^{\infty} \frac{d \mu(t)}{t+1}<\infty
$$

We also derive norm estimates for the difference $h_{\mu}(A)-h_{\mu}(\tilde{A})$.

The considered functions belong to the class of so called Hirsch functions [9]. The important examples here are the operator fraction powers and operator logarithm. The theories of the fraction powers and logarithm are well developed. For the classical results, see $[8,9]$. The recent investigations can be found in $[1,2,4,5],[10]-[15]$ and the references given therein.

At the same time, to the best of our knowledge, the norm estimates and perturbation results for these operator functions are derived mainly in the case of self-adjoint operators, cf. [3]. Below we do not require that $A$ and $\tilde{A}$ are self-adjoint.

Put $A_{I}:=\left(A-A^{*}\right) / 2 i$ and $A_{R}\left(A+A^{*}\right) / 2$ and assume that

$$
\beta(A):=\inf \operatorname{Re} \sigma(A)>0 .
$$

It is also supposed that

$$
D\left(A^{*}\right)=D(A), A_{I} \text { is bounded, } A_{R} \text { is invertible and } A_{R}^{-1} \in \mathcal{S}_{2} .
$$

Denote by $a_{k}$ the eigenvalues of $A$ with their multiplicities taken into account and enumerated in the non-decreasing order of their absolute values: $\left|a_{k}\right| \leq\left|a_{k+1}\right|(k=1,2, \ldots)$.

We will check that conditions (1.1), (1.2) yield that

$$
\tau(A):=\left[\sum_{k=1}^{\infty} \frac{1}{\left|a_{k}\right|^{2}}\right]^{1 / 2}<\infty .
$$

Put

$$
\phi(x):=\sum_{k=0}^{\infty} \frac{x^{k}}{\sqrt{k !}}(x \geq 0) .
$$

Now we are in a position to formulate the main result of this paper. 
Theorem 1. Let conditions (1.1), (1.2) hold. Then

$$
\left\|h_{\mu}(A)\right\| \leq \phi\left(2\left\|A_{I}\right\| \tau(A)\right) J(A, \mu),
$$

where

$$
J(A, \mu):=\int_{0}^{\infty} \frac{d \mu(t)}{t+\beta(A)}
$$

The proof of this theorem is divided into a series of lemmas, which are presented in the next two sections.

If $A$ is selfadjoint, then $\phi\left(2\left\|A_{I}\right\| \tau(A)\right)=\phi(0)=1$ and by Theorem 1 $\left\|h_{\mu}(A)\right\| \leq J(A, \mu)$. Note that by the Schwarz inequality,

$$
\sum_{k=0}^{\infty} \frac{x^{k}}{(k !)^{1 / 2}}=\sum_{k=0}^{\infty} \frac{(\sqrt{2} x)^{k}}{(\sqrt{2})^{k}(k !)^{1 / 2}} \leq\left(\sum_{j=0}^{\infty} \frac{2^{j} x^{2 j}}{j !} \sum_{k=0}^{\infty} \frac{1}{2^{k}}\right)^{1 / 2}=\sqrt{2} e^{x^{2}} .
$$

Thus

$$
\phi(x) \leq \sqrt{2} e^{x^{2}}(x \geq 0) .
$$

In addition,

$$
\phi(x)=1+x \sum_{k=1}^{\infty} \frac{x^{k-1}}{\sqrt{k !}} \leq 1+x \sum_{k=1}^{\infty} \frac{x^{k-1}}{\sqrt{(k-1) !}}=1+x \phi(x)
$$

and consequently,

$$
\phi(x) \leq 1+\sqrt{2} x e^{x^{2}} \quad(x \geq 0) .
$$

For $x \leq 1-1 / \sqrt{2},(1.5)$ is sharper than (1.4).

Theorem 1, (1.4) and (1.5) imply

$$
\left\|h_{\mu}(A)\right\| \leq \sqrt{2} J(A, \mu) \exp \left[4\left\|A_{I}\right\|^{2} \tau^{2}(A)\right]
$$

and

$$
\left\|h_{\mu}(A)\right\| \leq J(A, \mu)\left(1+2 \sqrt{2}\left\|A_{I}\right\| \tau(A) \exp \left[4\left\|A_{I}\right\|^{2} \tau^{2}(A)\right]\right) .
$$

\section{Existence of the Schur basis}

Recall the Keldysh theorem, cf. [7, Theorem V. 8.1].

Theorem 2. Let $B=K_{0}\left(I+K_{1}\right)$, where $K_{0}=K_{0}^{*} \in \mathcal{S}_{r}$ for some $r \in[1, \infty)$ and $K_{1}$ is compact. In addition, let from $B f=0(f \in \mathcal{H})$ it follows that $f=0$. Then $B$ has a complete in $\mathcal{H}$ system of the root vectors. 
Note that due to (1.1), $A$ is boundedly invertible.

Lemma 3. Under conditions (1.1) and (1.2) the operator $A^{-1}$ has a complete system of root vectors and $A^{-1} \in \mathcal{S}_{2}$. But

Proof. Since $A=A_{R}+i A_{I}=\left(I+i A_{I} A_{R}^{-1}\right) A_{R}$, we have $I+i A_{I} A_{R}^{-1}=A A_{R}^{-1}$.

$$
\left(A A_{R}^{-1}\right)^{-1}=A_{R} A^{-1}=\left(A-i A_{I}\right) A^{-1}=I-i A_{I} A^{-1} .
$$

Since $A_{I}$ is bounded, operator $I+i A_{R}^{-1} A_{I}$ is boundedly invertible. We have

$$
A^{-1}=A_{R}^{-1}\left(I+i A_{I} A_{R}^{-1}\right)^{-1} \in \mathcal{S}_{2} .
$$

In addition, $\left(I+i A_{I} A_{R}^{-1}\right)^{-1}=I+K_{2}$, where

$$
K_{2}=\left(I+i A_{I} A_{R}^{-1}\right)^{-1}-I=-i A_{I} A_{R}^{-1}\left(I+i A_{I} A_{R}^{-1}\right)^{-1} \in \mathcal{S}_{2} .
$$

So $A^{-1}=A_{R}^{-1}\left(I+K_{2}\right)$, and from $A^{-1} f=0(f \in \mathcal{H})$ it follows that $f=0$. Now the Keldysh theorem implies the required result.

Lemma 4. Let an operator $A$ on $\mathcal{H}$ have a compact resolvent, and for some $b \notin \sigma(A),(A-b I)^{-1}$ have a complete system of root vectors. Then there is an orthogonal normal (Schur) basis $\left\{e_{k}\right\}_{k=1}^{\infty}$, in which $A$ is representable by a triangular matrix $\left(a_{j k}\right)_{1 \leq j \leq k \leq \infty}$ :

$$
A e_{k}=\sum_{j=1}^{k} a_{j k} e_{j} \text { and }\left\langle A e_{k}, e_{k}\right\rangle=a_{k} \quad(k=1,2, \ldots),
$$

where $a_{k}(k=1,2, \ldots)$ are the eigenvalues of $A$.

Proof. Put $M=A-b I$. Due to [7, Lemma I.4.1], there is an orthonormal basis $\left\{e_{k}\right\}_{k=1}^{\infty}$, in which $M^{-1}$ is represented by a triangular matrix $\left(d_{j k}\right)_{1 \leq j \leq k \leq \infty}$ :

$$
M^{-1} e_{k}=\sum_{j=1}^{k} d_{j k} e_{j} \quad(k=1,2, \ldots) .
$$

Due to the spectrum mapping theorem, $d_{k k}=1 /\left(a_{k}-b\right)$ are the eigenvalues of $M^{-1}$. Denote

$$
P_{k}=\sum_{j=1}^{k}\left\langle., e_{j}\right\rangle e_{j} \quad(k=1,2, \ldots) ; P_{0}=0 .
$$


Then

$$
M^{-1} P_{k}=P_{k} M^{-1} P_{k}(k=1,2, \ldots) .
$$

Besides,

$$
\Delta P_{k} M^{-1} \Delta P_{k}=\frac{1}{a_{k}-b} \Delta P_{k}\left(\Delta P_{k}=P_{k}-P_{k-1} ; k=1,2, \ldots\right) .
$$

Let us check that

$$
M P_{k} f=P_{k} M P_{k} f(k=1,2, \ldots ; f \in D(A)) .
$$

Indeed, $M^{-1} P_{k}$ is an invertible $k \times k$ matrix, and therefore, $M^{-1} P_{k} \mathcal{H}$ is dense in $P_{k} \mathcal{H}$. Since $\Delta P_{j} P_{k}=0$ for $j>k$, we have $0=\Delta P_{j} M M^{-1} P_{k}=\Delta P_{j} M P_{k} M^{-1} P_{k}$. Hence $\Delta P_{j} M f=0$ for any $f \in P_{k} \mathcal{H}$. So (2.4) is valid and $\Delta P_{j} M e_{k}=0$ for $k<j$. Hence, $\Delta P_{j} M=\Delta P_{j} M\left(I-P_{j-1}\right)$, and

$$
\begin{gathered}
\Delta P_{j}=\Delta P_{j} \Delta P_{j}=\Delta P_{j} M M^{-1} \Delta P_{j}=\Delta P_{j} M\left(I-P_{j-1}\right) P_{j} M^{-1} \Delta P_{j} \\
=\Delta P_{j} M \Delta P_{j} M^{-1} \Delta P_{j}=\Delta P_{j} M \Delta P_{j} d_{j j} .
\end{gathered}
$$

So

$$
\Delta P_{j} M \Delta P_{j}=\frac{1}{d_{j j}} \Delta P_{j}=\left(a_{j}-b\right) \Delta P_{j} .
$$

From (2.4) and (2.5) it follows

$$
A P_{k} f=P_{k} A P_{k} f(k=1,2, \ldots ; f \in D(A))
$$

and

$$
\Delta P_{j} A \Delta P_{j}=a_{j} \Delta P_{j} \quad(j=1,2, \ldots) .
$$

This implies the required result.

From Lemmas 3 and 4 it follows

Corollary 5. Let conditions (1.1) and (1.2) hold. Then there is an orthogonal normal basis $\left\{e_{k}\right\}_{k=1}^{\infty}$, such that (2.1) is valid, and therefore, $A=S+W$, where $S e_{k}=a_{k} e_{k}(k=1,2, \ldots)$ (the diagonal part of $A$ ) and $W$ is defined by

$$
W e_{k}=\sum_{j=1}^{k-1} e_{j} a_{j k} \quad(k=2,3, \ldots),
$$

Therefore, $\sigma(S)=\sigma(A)=\left\{a_{k}\right\}$ and by the Weyl inequalities [7, Section II.3]

$$
\sum_{k=1}^{\infty} \frac{1}{\left|a_{k}\right|^{2}} \leq N_{2}^{2}\left(A^{-1}\right)
$$

and consequently, $S^{-1} \in \mathcal{S}_{2}$. 


\section{Proof of Theorem 1}

Put

$$
S_{I}:=\left(S-S^{*}\right) / 2 i=\sum_{j=1}^{\infty}\left(\operatorname{Im} a_{j}\right) \Delta P_{j}
$$

and $W_{I}=A_{I}-S_{I}=\left(W-W^{*}\right) / 2 i$. Since $P_{k-1} W P_{k}=W P_{k}$, it is not hard to check that $\left\langle W e_{k}, e_{k}\right\rangle=0$. In addition, $\left\langle A e_{k}, e_{k}\right\rangle=\left\langle S e_{k}, e_{k}\right\rangle=a_{k}$, and we have $\left\langle A_{I} e_{k}, e_{k}\right\rangle=\left\langle S_{I} e_{k}, e_{k}\right\rangle=\operatorname{Im} a_{k}$. So $\left|\operatorname{Im} a_{k}\right| \leq\left\|A_{I}\right\|$ and $\left\|S_{I}\right\|=\sup _{k}\left|\operatorname{Im} a_{k}\right|$ $\leq\left\|A_{I}\right\|$.

Furthermore, note that $P_{k-1} W^{*} P_{k}=0$ and thus $P_{k-1} W^{*} e_{k}=0$, and

$$
\begin{gathered}
\left\|W e_{k}\right\|=\left\|P_{k-1} W e_{k}\right\|=\left\|P_{k-1}\left(W-W^{*}\right) e_{k}\right\| \leq\left\|\left(W-W^{*}\right) e_{k}\right\| \\
=2\left\|W_{I} e_{k}\right\| .
\end{gathered}
$$

With $c_{k}=\operatorname{Im} a_{k}$ we have

$$
\begin{aligned}
\left\langle A_{I} e_{k}, A_{I} e_{k}\right\rangle= & \left\langle\left(W_{I}+c_{k}\right) e_{k},\left(W_{I}+c_{k}\right) e_{k}\right\rangle=\left\langle W_{I} e_{k}, W_{I} e_{k}\right\rangle \\
& +c_{k}\left\langle W_{I} e_{k}, e_{k}\right\rangle+c_{k}\left\langle e_{k}, W_{I} e_{k}\right\rangle .
\end{aligned}
$$

But $\left\langle W_{I} e_{k}, e_{k}\right\rangle=0$ and therefore $\left.\left.\left\langle A_{I} e_{k}, A_{I} e_{k}\right\rangle=\right\rangle W_{I} e_{k}, W_{I} e_{k}\right\rangle+c_{k}^{2}$. Consequently,

$$
\left\|W e_{k}\right\|^{2} \leq 4\left\|W_{I} e_{k}\right\|^{2} \leq 4\left(\left\|A_{I} e_{k}\right\|^{2}-\left|\operatorname{Im} a_{k}\right|^{2}\right) .
$$

Hence,

$$
\begin{gathered}
N_{2}^{2}\left(W S^{-1}\right)=\sum_{k=1}^{\infty}\left\|W S^{-1} e_{k}\right\|^{2}=\sum_{k=1}^{\infty} \frac{1}{\left|a_{k}\right|^{2}}\left\|W e_{k}\right\|^{2} \\
\leq \sum_{k=1}^{\infty} \frac{1}{\left|a_{k}\right|^{2}} \sup _{k}\left\|W e_{k}\right\|^{2} \leq 4\left\|A_{I}\right\|^{2} N_{2}^{2}\left(S^{-1}\right) .
\end{gathered}
$$

Lemma 6. Let conditions (1.1) and (1.2) hold. Then

$$
\left\|(A-z I)^{-1}\right\| \leq \frac{1}{d(A, z)} \phi\left(2\left\|A_{I}\right\| N_{2}\left((S-z I)^{-1}\right)\right) \quad(z \notin \sigma(A)),
$$

where $d(A, z)=\inf _{k}\left|a_{k}-z\right|$.

Proof. Making use of Corollary 5, we obtain $A-Z=S+W-z I=$ $\left(I-B_{z}\right)(S-z I)$, where $B_{z}:=-W(S-I z)^{-1}(z \notin \sigma(S)=\sigma(A))$. Since $W$ is 
bounded and $(S-z)^{-1} \in \mathcal{S}_{@}$, we have $B_{z} \in \mathcal{S}_{2}$. Take into that $P_{k-1} B_{z} P_{k}=$ $B_{z} P_{k}$, and thus $B_{z}$ is the triangular compact matrix with the zero diagonal. Therefore it is the limit of nilpotent matrices in the operator norm. Hence, due to [7, Theorem I.4.1], $B_{z}$ is quasi-nilpotent, and we can write

$$
\left(I-B_{z}\right)^{-1}=\sum_{k=0}^{\infty} B_{z}^{k}
$$

So,

$$
\left\|(I z-A)^{-1}\right\| \leq\left\|(S-I z)^{-1}\right\|\left\|\left(I-B_{z}\right)^{-1}\right\| \leq\left\|(S-I z)^{-1}\right\| \sum_{k=0}^{\infty}\left\|B_{z}^{k}\right\| .
$$

Due to [6, Corollary 7.4], for any $K \in \mathcal{S}_{2}$.

$$
\left\|K^{k}\right\| \leq \frac{N_{2}^{k}(K)}{(k !)^{1 / 2}} \quad(k=1,2, \ldots) .
$$

Making use of this inequality, we get

$$
\left\|B_{z}^{k}\right\| \leq \frac{N_{2}^{k}\left(B_{z}\right)}{(k !)^{1 / 2}}(k=1,2, \ldots) .
$$

But by (3.2)

$$
\begin{gathered}
N_{2}^{2}\left(B_{z}\right)=N_{2}^{2}\left(W(S-z I)^{-1}\right)=\sum_{k=1}^{\infty}\left\|W(S-z)^{-1} e_{k}\right\|^{2} \\
=\sum_{k=1}^{\infty} \frac{1}{\left|a_{k}-z\right|^{2}}\left\|W e_{k}\right\|^{2} \\
\leq \sum_{k=1}^{\infty} \frac{1}{\left|a_{k}-z\right|^{2}} \sup _{k}\left\|W e_{k}\right\|^{2} \leq 4\left\|A_{I}\right\|^{2} N_{2}^{2}\left((S-z I)^{-1}\right) .
\end{gathered}
$$

Thus

$$
\left\|B_{z}^{k}\right\| \leq \frac{2\left\|A_{I}\right\|^{k} N_{2}^{k}\left((S-z I)^{-1}\right)}{(k !)^{1 / 2}}(k=1,2, \ldots) .
$$

Moreover,

$$
\left\|(S-z I)^{-1}\right\|=\frac{1}{d(A, z)},
$$

Now (3.3) implies the required result. 
If $A$ is self-adjoint, then by Lemma 6

$$
\left\|(A-z I)^{-1}\right\| \leq \frac{1}{d(A, z)}
$$

$(z \notin \sigma(A))$.

Furthermore, since $\frac{1}{a_{k}-z}$ are the eigenvalues of $(A-z I)^{-1}$, making use of the Weyl inequalities, we have

$$
N_{2}^{2}\left((S-z I)^{-1}\right)=\sum_{k=1}^{\infty} \frac{1}{\left|a_{k}-z\right|^{2}} \leq N_{2}^{2}\left((A-z I)^{-1}\right) \quad(z \notin \sigma(A))
$$

Let $\operatorname{Re} a_{k}=b_{k}$ and $\operatorname{Im} a_{k}=c_{k}$. Then for any $t \geq 0, d(A,-t) \geq \beta(A)+t$ and

$$
\left|a_{k}+t\right|^{2}=\left(b_{k}+t\right)^{2}+c_{k}^{2}=\geq\left(b_{k}^{2}+2 b_{k} t+t^{2}+c_{k}^{2}=\left|a_{k}\right|^{2}+2 b_{k} t+t^{2} .\right.
$$

Hence, $N_{2}\left((S+t)^{-1}\right)=\tau(A, t)$, where

$$
\tau(A, t):=\left(\sum_{k=1}^{\infty} \frac{1}{\left|a_{k}\right|^{2}+2 b_{k} t+t^{2}}\right)^{1 / 2} \leq \tau(A, 0)=\tau(A) .
$$

Thus Lemma 6 yields

Corollary 7. Let conditions (1.1), (1.2) hold. Then

$$
\begin{gathered}
\left\|(A+t I)^{-1}\right\| \leq \frac{1}{(t+\beta(A))} \phi\left(2\left\|A_{I}\right\| \tau(A, t)\right) \\
\leq \frac{1}{(t+\beta(A))} \phi\left(2\left\|A_{I}\right\| \tau(A)\right) \quad(t \geq 0) .
\end{gathered}
$$

and therefore,

$$
\left\|h_{\mu}(A)\right\| \leq \int_{0}^{\infty} \frac{\phi\left(2\left\|A_{I}\right\| \tau(A, t)\right)}{t+\beta(A)} d \mu(t)
$$

Proof of Theorem 1. The assertion of the theorem follows from Corollary 7 and (3.5). 


\section{Perturbations of the Hirsch type functions}

Let $\tilde{A}$ and $A$ be linear operators on $\mathcal{H}$ with the same dense domain:

$$
D(\tilde{A})=D(A) \text { and } q:=\|A-\tilde{A}\|<\infty .
$$

Lemma 8. Let the conditions (4.1),

$$
\left\|(A+t)^{-1}\right\| \leq \frac{m_{1}}{1+t}\left(m_{1}=\text { const }, t \geq 0\right)
$$

and

$$
q m_{1}<1
$$

hold. Then

$$
\left\|h_{\mu}(\tilde{A})\right\| \leq \frac{m_{1}}{1-q m_{1}} \int_{0}^{\infty} \frac{d \mu(t)}{1+t}
$$

and

$$
\left\|h_{\mu}(\tilde{A})-h_{\mu}(A)\right\| \leq \frac{q m_{1}^{2}}{1-q m_{1}} \int_{0}^{\infty} \frac{d \mu(t)}{(1+t)^{2}}
$$

Proof. Since $\left\|(A+I t)^{-1}\right\| \leq m_{1}(t \geq 0)$, by the Hilbert identity

$$
(\tilde{A}+I t)^{-1}-(\tilde{A}+I t)^{-1}=(\tilde{A}+I t)^{-1}(\tilde{A}+I t)^{-1}(A-\tilde{A})(\tilde{A}+I t)^{-1}
$$

and (4.3) we get

$$
\left\|(\tilde{A}+I t)^{-1}\right\| \leq \frac{\left\|(A+I t)^{-1}\right\|}{1-q\left\|(A+I t)^{-1}\right\|} \leq \frac{\left\|(A+I t)^{-1}\right\|}{1-q m_{1}}(t \geq 0) .
$$

Hence, condition (4.2) implies

$$
\left\|(\tilde{A}+I t)^{-1}\right\| \leq \frac{m_{1}}{\left(1-q m_{1}\right)(1+t)} .
$$

Moreover, by (4.4)

$$
\left\|(\tilde{A}+I t)^{-1}-(A+I t)^{-1}\right\| \leq \frac{q m_{1}^{2}}{\left(1-q m_{1}\right)(1+t)^{2}} .
$$

Consequently,

$$
\left\|h_{\mu}(\tilde{A})\right\| \leq \int_{0}^{\infty}\left\|(\tilde{A}+i t)^{-1}\right\| d \mu(t) \leq m_{1} \int_{0}^{\infty} \frac{d \mu(t)}{\left(1-q m_{1}\right)(1+t)}
$$

and

$$
\left\|h_{\mu}(\tilde{A})-h_{\mu}(A)\right\| \leq \int_{0}^{\infty}\left\|(A+i t)^{-1}-(\tilde{A}+i t)^{-1}\right\| d \mu(t)
$$




$$
\leq \frac{q m_{1}^{2}}{1-q m_{1}} \int_{0}^{\infty} \frac{d \mu(t)}{(1+t)^{2}}
$$

as claimed.

Let conditions (1.1), (1.2) hold. Then from Corollary 7 it follows that

$$
\left\|(A+I t)^{-1}\right\| \leq \phi\left(2\left\|A_{I}\right\| \tau(A)\right) \frac{1}{\beta(A)+t} \leq \frac{m_{0}(A)}{1+t},
$$

where

$$
m_{0}(A)=\phi\left(2\left\|A_{I}\right\| \tau(A)\right) \sup _{t \geq 0} \frac{1+t}{\beta(A)+t} .
$$

Simple calculations show that

$$
m_{0}(A)=\phi_{\mu}\left(2\left\|A_{I}\right\| \tau(A)\right) \begin{cases}1 & \text { if } \beta(A)>1 \\ \frac{1}{\beta(A)} & \text { if } \beta(A) \leq 1 .\end{cases}
$$

Now Lemma 8 implies

Corollary 9. Let the conditions (1.1), (1.2), (4.1) and $q m_{0}(A)<1$ hold. Then,

$$
\left\|h_{\mu}(\tilde{A})\right\| \leq \frac{m_{0}(A)}{1-q m_{0}(A)} \int_{0}^{\infty} \frac{d \mu(t)}{1+t}
$$

and

$$
\left\|h_{\mu}(\tilde{A})-h_{\mu}(A)\right\| \leq \frac{q m_{0}^{2}(A)}{1-q m_{0}(A)} \int_{0}^{\infty} \frac{d \mu(t)}{(1+t)^{2}} .
$$

\section{Fractional powers}

Recall that a closed densely defined operator $A$ is called a non-negative one, if $(-\infty, 0)$ is included in the resolvent set and $\sup _{t>0}\left\|t(A+t)^{-1}\right\|<\infty$, cf. [9, p. 1]. For a closed non-negative operator $A$, the negative fractional power can be a defined by the formula

$$
A^{-\nu}=\frac{\sin (\pi \nu)}{\pi} \int_{0}^{\infty} t^{-\nu}(A+I t)^{-1} d t \quad(0<\nu<1),
$$

cf. [9]. Under condition (4.2) $A$ is non-negative. 
If conditions (1.1), (1.2) hold, then due to Corollary 3.2, $A$ is non-negative and by Theorem 1

$$
\begin{aligned}
\left\|A^{-\nu}\right\| \leq & \frac{\sin (\pi \nu)}{\pi} \phi\left(2\left\|A_{I}\right\| \tau(A)\right) \int_{0}^{\infty} \frac{t^{-\nu} d t}{t+\beta(A)} \\
& \leq m_{0}(A) \frac{\sin (\pi \nu)}{\pi} \int_{0}^{\infty} \frac{t^{-\nu} d t}{1+t}
\end{aligned}
$$

If, in addition, the conditions (4.1) and $q m_{0}(A)<1$ are fulfilled, then by Corollary 4.2,

$$
\left\|\tilde{A}^{-\nu}\right\| \leq \frac{m_{0}(A)}{1-q m_{0}(A)} \int_{0}^{\infty} \frac{t^{-\nu} d t}{1+t}
$$

and

$$
\left\|\tilde{A}^{-\nu}-A^{-\nu}\right\| \leq \frac{q m_{0}^{2}(A)}{1-q m_{0}(A)} \int_{0}^{\infty} \frac{t^{-\nu} d t}{(1+t)^{2}}
$$

\section{Logarithm}

By [9, formula (10.3), p. 245] for a non-negative operator with a dense range $R(A)$, we can define the logarithm by

$$
\log (A) y=\int_{0}^{\infty}(t+A)^{-1}(A y-y) \frac{d t}{t+1} \quad(y \in D(A) \cap R(A))
$$

(see also [9, p. 246, line -7]).

Let conditions (1.1), (1.2) hold. Then by Theorem 1

$$
\left\|\int_{0}^{\infty}(t+A)^{-1} \frac{d t}{t+1}\right\| \leq \phi\left(2\left\|A_{I}\right\| \tau(A)\right) J_{l}
$$

where

$$
J_{l}(A):=\int_{0}^{\infty} \frac{d t}{(t+\beta(A))(t+1)} \leq m_{0}(A) \int_{0}^{\infty} \frac{d t}{(t+1)^{2}}=m_{0}(A) .
$$

Since $A$ is boundedly invertible we have $R(A)=\mathcal{H}$. Now (6.1) implies

$$
\begin{gathered}
\|\log (A) y\| \leq \phi\left(2\left\|A_{I}\right\| \tau(A)\right) J_{l}\|A y-y\| \leq m_{0}(A)\|A y-y\| \\
(y \in D(A)),
\end{gathered}
$$


provided conditions (1.1), (1.2) hold. Furthermore, according to (6.1),

$$
\begin{aligned}
& \log (\tilde{A}) y-\log (A) y=\int_{0}^{\infty}\left[(t+\tilde{A})^{-1}(\tilde{A} y-y)-(t+A)^{-1}(\tilde{A} y-y)\right] \frac{d t}{t+1} \\
= & \int_{0}^{\infty}\left[(t+\tilde{A})^{-1}-(t+A)^{-1}\right] \frac{d t}{t+1}(\tilde{A} y-y)+\int_{0}^{\infty}(t+A)^{-1} \frac{d t}{t+1}(\tilde{A}-A) y .
\end{aligned}
$$

So

$$
\begin{gathered}
\left.\|\log (\tilde{A}) y-\log (A) y\| \leq\left\|\int_{0}^{\infty}\left[(t+\tilde{A})^{-1}-(t+A)^{-1}\right] \frac{d t}{t+1}\right\| \| \tilde{A} y-y\right) \| \\
+\left\|\int_{0}^{\infty}(t+A)^{-1} \frac{d t}{t+1}\right\|\|(A-\tilde{A}) y\| .
\end{gathered}
$$

Making use of Corollary 4.2, we can assert that

$$
\begin{aligned}
& \|\log (\tilde{A}) y-\log (A) y\| \leq \frac{q m_{0}^{2}(A)}{\left(1-q m_{0}(A)\right)} \int_{0}^{\infty} \frac{d t}{(t+1)^{3}}\|\tilde{A} y-y\| \\
& +q m_{0}(A)\|y\|=\frac{q m_{0}^{2}(A)}{2\left(1-q m_{0}(A)\right)}\|\tilde{A} y-y\|+q m_{0}(A)\|y\|,
\end{aligned}
$$

provided the conditions (1.1), (1.2), (4.1) and $q m_{0}(A)<1$ hold.

\section{References}

[1] H. Al Baba, Fractional powers of the Stokes operator with boundary conditions involving the pressure. Math. Nachr., 292, No 6 (2019), 1194-1212.

[2] A. Ashyralyev and A. Hamad, A note on fractional powers of strongly positive operators and their applications. Fract. Calc. Appl. Anal. 22, No 2 (2019), 302-325.

[3] M.Sh. Birman and M.Z. Solomyak, Estimates for the difference of fractional powers of selfadjoint operators in the case of unbounded perturbations, $\mathrm{Za}$ piski Nauchnykh Seminarov Leningradskogo Otdeleniya Matemaficheskogo Instituta im. V. A. Steklova Akademii Nauk SSSR, 178 (1989), 120-145 (In Russian).

[4] O. Ciaurri, L. Roncal and S. Thangavelu, Hardy-type inequalities for fractional powers of the Dunkl-Hermite operator, Proc. of the Edinburgh Mathematical Society, 61 (2018), 513-544. 
[5] S. Clark, Sums of operator logarithms, Quart. J. Math. 60 (2009), 413-427.

[6] M.I. Gil', Operator Functions and Operator Equations, World Scientific, New Jersey (2018).

[7] I.C. Gohberg, and M.G. Krein, Introduction to the Theory of Linear Nonselfadjoint Operators, Trans. Mathem. Monographs, Vol. 18, Amer. Math. Soc., Providence, R.I. (1969).

[8] M. Haase, The Functional Calculus for Sectorial Operators, Birkhäuser Verlag (2006).

[9] C. Martínez and M. Sanz, The Theory of Fractional Powers of Operators, North-Holland Mathematics Studies, 187. Elsevier, Amsterdam (2001).

[10] J. Pastor, On uniqueness of fractional powers of multi-valued linear operators and the incomplete Cauchy problem, Annali di Matematica, 191 (2012), 167-180.

[11] A. Prasad and K. Mahato, Two versions of fractional powers of Hankeltype transformations and pseudo-differential operators, Rend. Circ. Mat. Palermo 65 (2016), 209-241.

[12] A. Prasad and P.K. Maurya, A couple of fractional powers of Hankeltype integral transformations and pseudo-differential operators, SeMA $\mathbf{7 4}$ (2017), 181-211.

[13] L. Silvestre, Regularity of the obstacle problem for a fractional power of the Laplace operator, Commun. Pure Appl. Math. 60, No 1 (2007), 67-112.

[14] C. Schmoeger, On logarithms of linear operators on Hilbert spaces. Demonstratio Math. 35, No 2, (2002), 375-384.

[15] Yang, Changsen and Zuo, Hongliang, A monotone operator function via Furuta-type inequality with negative powers, Math. Inequal. Appl. 6, No 2 (2003), 303-308. 
